\title{
Fully-automated E-field measurement system using pigtailed electro-optic sensors for temperature- dependent-free measurements of microwave signals in outdoors conditions
}

\author{
M. Bernier ${ }^{1}$, L. Duvillaret ${ }^{1}$, G. Gaborit ${ }^{2}$, A. Paupert ${ }^{3}$, J.-L. Lasserre ${ }^{3}$ \\ ${ }^{1}$ IMEP-LAHC, UMR CNRS 5130, 3 parvis Louis Néel, 38016 Grenoble Cedex 1, France \\ ${ }^{2}$ IMEP-LAHC, UMR CNRS 5130, Université de Savoie, Campus Scientifique, 73376 Le Bourget du Lac Cedex, France \\ ${ }^{3}$ DGA/DET/Centre d'Etude de Gramat/SDP/CGN/EXC, 46500 Gramat, France
}

\begin{abstract}
We present, in this paper, pigtailed electro-optic sensors dedicated to the measurement of high power microwave signals in outdoors conditions. We give results on high power microwave single-shot pulses obtained with long fibre links (> $20 \mathrm{~m}$ ) in hard environmental conditions (temperature variations up to $30^{\circ} \mathrm{C}$ ). In these awkward outdoors conditions we obtain very stable measurements with $\mathrm{rms}$ signal fluctuations lower than $0.2 \mathrm{~dB}$. We focus here especially on the design of the temperature-dependent-free system.
\end{abstract}

\section{INTRODUCTION}

Since the 80's, studies [2] have demonstrated the great potentiality of the linear electro-optic (EO) effect to probe electric (E) fields. Firstly dedicated to the characterization of guided waves and especially MMICs [3], these sensors, based on the E-field induced variation of the refractive index of an EO crystal, are also perfectly suited for ultra wide band, radiated E field measurements [4]. We have developed fully dielectric probes presenting a very low invasiveness, a higher selectivity (measurement of a unique E-field component) and much higher spatial and temporal resolutions than classical sensors based on antennas. These probes allow performing very accurate vectorial $\mathrm{E}$ field mapping of either repetitive or single-shot signals in the far- and near-field regions. Their major drawbacks concern, on the one hand, their rather low sensitivity in comparison with antennas [5], and, on the other hand, the temporal instability of their EO response unless the measurements are performed within a temperature-stabilized room. The development of competitive and versatile EO transducers dedicated to microwave E-field measurements in outdoors conditions requires then to deal first with the temperature dependence of the EO sensors response. More

\footnotetext{
* An earlier version of this paper [1] was presented at the 2007 IEEE SENSORS Conference and was published in its proceedings.
}

generally, thermal effects will inevitably affect, at different levels, every sensors dedicated to measure any physical quantity but the temperature, or devices using the non linear properties of media (e.g. electro- and acousto-optic modulators) [6]. Different solutions have been explored to get rid of these detrimental effects. Some of them are aimed at developing EO materials whose EO coefficients are insensitive to temperature variations over a wide temperature range [7] but with remaining temperature dependence of the birefringence. In our case, we decide to compensate, rather than eliminate, the thermal effect induced by temperature variations. The compensation technique, presented in this paper, presents the key advantage to be adaptable to any EO transducer based on the polarization state modulation, regardless the EO crystal constituting the probe tip. Therefore, it permits to develop cost effective probes using common EO crystals $\left(\mathrm{LiNbO}_{3}\right.$ and $\mathrm{LiTaO}_{3}$ ). It is also time efficient in terms of development and realization, and moreover, permits to get information on the temperature of the EO crystal [8].

This paper presents a servo-assisted system ensuring a four-parameters real-time control that allows performing reliable outdoors vectorial (magnitude and phase) measurements of microwave signals. The fully automated optical bench developed, and its associated $\mathrm{LiTaO}_{3} \mathrm{EO}$ probe, permit to stabilize the E-field response, compensating the detrimental effects induced by temperature variations, whatever part of the transducer on which they apply. Therefore, the proposed solution answers users' expectations desirous to get accurate and reliable vectorial measurement of high power microwave (HPM) E fields. Compared to antennas [9], the pigtailed probe presents a flat response over a much wider frequency bandwidth ( $\sim 8$ decades $)$ and permits a metal-free remote access (several tens of meters) to one E-field component and the temperature inside the EO crystal . 
The paper is divided in three parts. In the first one we discuss about the principle of the EO detection we use to measure HPM E fields and the problems caused by temperature changes. In the second part, we expose the improvements made on the optical bench and the EO probe to compensate those detrimental thermal effects. We present results validating the solutions we have brought in the third part.

\section{PRINCIPLE}

The measurement bench (cf. Fig. 1) uses the modulation of the polarization state of a laser probe beam $(\lambda=1550 \mathrm{~nm})$ induced by a microwave E field applied to the EO crystal. This polarization state modulation is converted into modulated optical powers, thanks to the Wollaston prism (element (3) in Fig. 1), which are then detected by the photodiodes $\mathrm{PD}_{1}$ and $\mathrm{PD}_{2}$. The microwave modulation of the optical powers $\mathrm{I}_{1}$ and $\mathrm{I}_{2}$ detected by the photodiodes $\mathrm{PD}_{1}$ and $\mathrm{PD}_{2}$, respectively, are directly proportional to the magnitude of the E field to be measured [10]. A linear response of the EO transducer with respect to the microwave signal is achievable if the optical probe beam at the entrance of the Wollaston prism, placed up stream $\mathrm{PD}_{1}$ and $\mathrm{PD}_{2}$ (element (3) in Fig. 1), is linearly polarized in absence of microwave $\mathrm{E}$ field, and oriented with an angle of $45^{\circ}$ about the eigen dielectric axes of the Wollaston prism [10]. For this welldefined working point the EO transducer presents also a maximum of its sensitivity. Therefore the EO response is kept constant as long as this optimal working point is maintained during time.

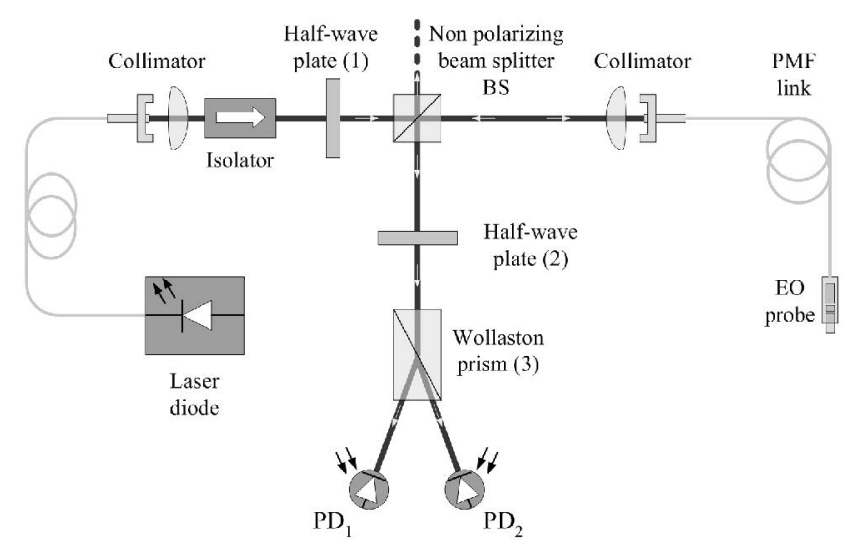

Fig. 1. Scheme of the setup for E-field measurement using EO sensors based on polarization state modulation.

For that purpose, a half-wave plate (element (1) in Fig. 1) is used to align a linearly polarized laser beam along one of the eigen dielectric axes of a polarizing maintaining fiber (PMF). Therefore, we get a linearly polarized laser beam at the entrance of the EO probe.Next, an embedded quarter wave plate, placed after the Grin lens (see Fig. 2), transforms the incoming polarization from linear into circular. In this way, the laser beam will equally probe the two eigen dielectric axes of the EO crystal, whatever its orientation $\alpha$ about the eigen dielectric axes of the PMF is.

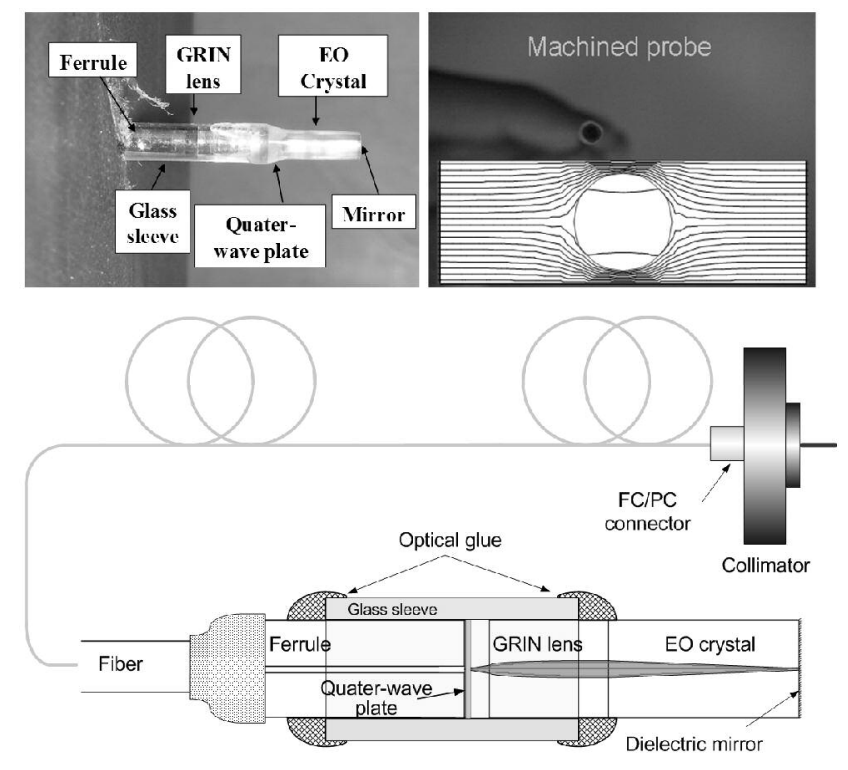

Fig. 2. Picture and scheme of the designed EO probe. Drawing on the top right picture presents also the simulated internal E-field with equipotential lines.

After propagating back and forth into the EO crystal, the laser beam is launched back within the PMF. The optical E field $\overrightarrow{\mathrm{E}}_{\mathrm{opt}}$ coming out from the PMF can be computed thanks to the Jones' formalism [11] and is given by:

$$
\vec{E}_{\text {opt }}=E_{0}\left(\begin{array}{l}
\sin \Delta \varphi \exp \left(-j \frac{\Delta \phi+2 \alpha}{2}\right) \\
\cos \Delta \varphi \exp \left(+j \frac{\Delta \phi+2 \alpha}{2}\right)
\end{array}\right)
$$

where $\Delta \varphi$ and $\Delta \phi$ are the relative phase shifts induced by the birefringence of the EO crystal and the PMF, respectively. The Jones' vector (1) clearly shows that the birefringence of both EO crystal and PMF are split up thanks to the embedded quarter-wave plate. Indeed, the magnitudes of each optical E-field component depend on the birefringence of the EO crystal, whereas the relative phase shift between them depends on the birefringence of the PMF and on the orientation $\alpha$ of the EO crystal. Fig. 3 represents the polarization state given by (1). On this figure the angle $\Delta \phi+2 \alpha$ is given by the intersection of a circle of radius $E_{0} \cos (\Delta \varphi)$ and the parallel to the $x$ axis that cuts the ellipse at its maximum along the $x$ axis. This angle is also given by the intersection of a circle of radius $E_{0} \sin (\Delta \varphi)$ and the parallel to the $y$ axis that cuts the ellipse at its maximum along the $y$ axis. Thus, any change of EO crystal birefringence $(\Delta \varphi)$ or of PMF birefringence $(\Delta \phi)$ leads to a polarization state variation consisting of simultaneous 
changes of orientation $\delta$ and ellipticity $\xi$ of the optical polarization state. $\delta$ is defined as the orientation of the major ellipse axis while $\xi$ is defined as the ratio between the lengths $b$ and $a$ of the semi minor and the semi major ellipse axes, respectively.

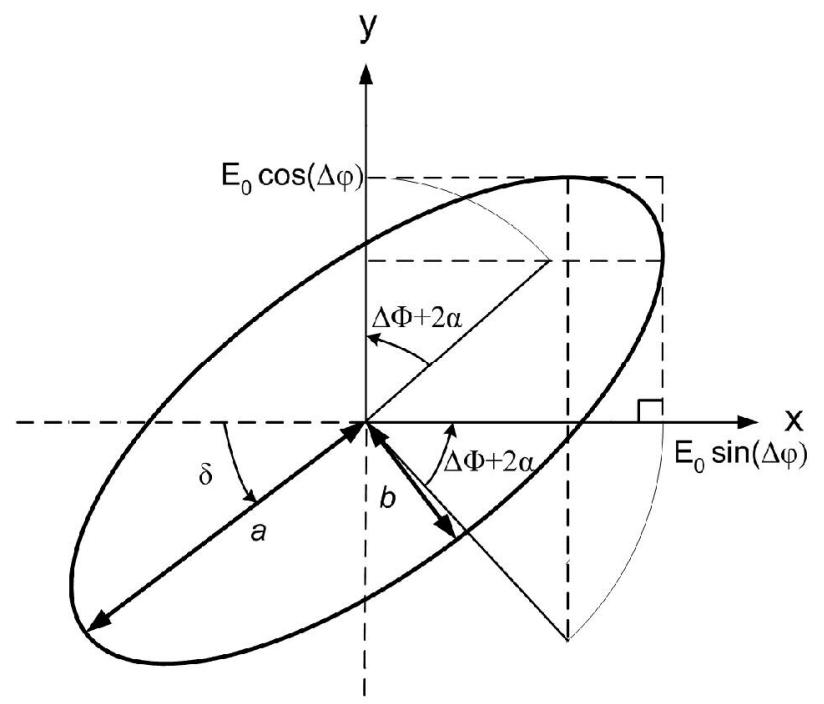

Fig. 3. Scheme of the polarization state coming out from the PMF and whose expression is given by (1).

The squares of the magnitudes of the E-field components along $x$ and $y$ axes are measured using the photodiodes $\mathrm{PD}_{1}$ and $\mathrm{PD}_{2}$ placed down stream the Wollaston prism. The optical powers received by $\mathrm{PD}_{1}$ and $\mathrm{PD}_{2}$ are written as:

$$
\left\{\begin{array}{l}
I_{1}=I_{0} \sin ^{2} \Delta \varphi \\
I_{2}=I_{0} \cos ^{2} \Delta \varphi
\end{array},\right.
$$

where $I_{0}$ represents the incident optical power.

On the other hand, the relative phase shift $\Delta \varphi$ is linked to the EO crystal length $L$, to its intrinsic birefringence $\Delta n_{0}$, and to the microwave E-field induced birefringence $\Delta n_{E}$ :

$$
\Delta \varphi=\underbrace{\frac{4 \pi L \Delta n_{0}}{\lambda}}_{\Delta \varphi_{0}}+\underbrace{\frac{4 \pi L \Delta n_{E}(\vec{E})}{\lambda}}_{\Delta \varphi_{E}}
$$

Consequently, the optical powers $I_{1}$ and $I_{2}$ are modified by the E field to be measured, thanks to the Pockels' effect [12]. Due to the weakness of the Pockels' effect, these optical powers can be approximated by their expansion into Taylor series to the second order:

$$
I_{2} \cong \frac{I_{0}}{2}(1 \mp \varepsilon)
$$

with $\varepsilon=\left(1-2 \Delta \varphi_{E}^{2}\right) \cos 2 \Delta \varphi_{0}-2 \Delta \varphi_{E} \sin 2 \Delta \varphi_{0}$. The Efield induced relative phase shift $\Delta \varphi_{E}$ can be expressed in term of sensitivity vector $\overrightarrow{\Delta K}$ [13]:

$$
\Delta \varphi_{E}=\frac{4 \pi L \overrightarrow{\Delta K} \cdot \vec{E}}{\lambda} .
$$

$\overrightarrow{\Delta K}$ depends on the orientation of the EO crystal and on direction of the laser probe beam wave vector. Looking at (4), one can see that the linear E-field dependence on optical powers $I_{1}$ and $I_{2}$ reaches its maximum whereas the quadratic E-field dependence vanishes for $\Delta \varphi_{0}=45^{\circ}$. Hence, this value defines the optimal working point for the system for which we get simultaneously the highest linearity and sensitivity. Then, $I_{1}$ and $I_{2}$ can be rewritten as:

$$
\underset{2}{I_{1}}(\vec{E}) \cong \frac{I_{0}}{2}\left(1 \pm \frac{8 \pi L}{\lambda} \overrightarrow{\Delta K} \cdot \vec{E}\right) .
$$

This latter set of equations clearly shows that the EO sensor presents a linear response with the E-field component parallel to $\overrightarrow{\Delta K}$. The sensitivity $S$, defined as the absolute value of the derivative of $I_{1,2}$ with respect to $\vec{E}$ (for $\vec{E} \overrightarrow{\Delta K}$ ), is then given by:

$$
S \cong I_{0} \frac{4 \pi L\|\overrightarrow{\Delta K}\|}{\lambda} .
$$

Let us remark that in the case of an EO probe without the quarter wave plate placed between the grin lens and the EO crystal, the optical powers detected are written as:

$$
\left\{\begin{array}{l}
I_{1} \cong I_{0}\left(1-\sin ^{2} 2 \alpha \sin ^{2} \Delta \varphi\right) \\
I_{2} \cong I_{0} \sin ^{2} 2 \alpha \sin ^{2} \Delta \varphi
\end{array} .\right.
$$

As shown in the set of Eq. (8), such EO probe does not permit to get simultaneous highest linearity and sensitivity without setting tricky conditions on the orientation of the EO crystal with respect to the eigen dielectric axes of the PMF. The addition of the embedded quarter wave plate simplifies the probe assembly and allows getting a system whose performances depend only on the orientation of the polarization state of the return optical beam at the entrance of the Wollaston prism regardless the orientation of the EO crystal.

The optimal working point of our system, defined by $\Delta \varphi_{0}=45^{\circ}$, is ensured by a half-wave plate, placed between the PMF and the Wollaston prism (element (3) in Fig. 1). It rotates the elliptically polarized probe beam until it reaches the right orientation. Unfortunately any temperature change of either the EO crystal [14] or its 
PMF link [15] generates birefringence variations that lead, in turn, to a drift of the working point.

Indeed, the ellipticity $\xi$ and the orientation $\delta$ of the polarization state of the optical probe beam, expressed in (1), are defined [16] by:

$$
\left\{\begin{array}{l}
\xi=\sqrt{\frac{1-\Lambda}{1+\Lambda}} \text { with } \Lambda^{2}=1-\sin ^{2} 2 \Delta \varphi \sin ^{2}(2 \alpha+\Delta \phi) \\
\delta=-\frac{1}{2} \arctan (\tan 2 \Delta \varphi \cos (2 \alpha+\Delta \phi))
\end{array} .\right.
$$

It means that any variation of either EO crystal birefringence or PMF birefringence, induced by a temperature change, modifies the polarization state orientation of the optical probe beam. Regarding the optimization condition, this orientation drift involves a partial loss of the useful information. Therefore, as the working point drift is directly linked to the PMF length via $\Delta \phi$, the longer the fiber link is, the less stable the system is. For example, a 10-meters long pigtailed probe using PANDA PMF prevents from obtaining reliable results on time durations exceeding a few seconds. For the same reason and considering Eq. (7), the longer the EO crystal is, the higher its sensitivity is but the less stable the EO transducer response is. In other words, for a given EO crystal, the sensitivity - measurement stability product is a constant.

In addition, the different interfaces between the constituting elements of the probe (see Fig. 2) lead to multiple reflections. Those Fabry-Pérot cavities lead in turn to parasitic modulations of the optical power $I_{0}$ reflected by the EO probe. Since the sensitivity $S$ of the EO probe is proportional to the return optical power $I_{0}$ (see Eq. (7)), it is then modified by any variation of the optical lengths of these parasitic Fabry-Pérot cavities, these latter ones depending on the temperature.

Then, regarding the principle of the EO transducer based on the polarization state modulation and considering the thermal effects on anisotropic EO crystals, it appears essential to lock the system on its optimal working point using a servo-controlled system. For that purpose, we have developed a four-parameters real-time servo-controlled system whose description is given in the following section.

\section{DESCRIPTION OF THE FOUR-PARAMETERS SERVO- CONTROLLED SYSTEM}

As we will see, the tracking of the optimal working point for the EO transducer requires additional optical elements with respect to the optical system presented in section II (cf. Fig. 1). Although the working point, previously defined, permits to get an optimized E-field measurement, it does not permit to distinguish the thermal effects affecting the EO crystal on the one hand, and the PMF link on the other hand. Nevertheless, it exists a specific polarization state for which the variation of its orientation, induced by the PMF temperature change, is nil. Indeed, such polarization state is defined by $\partial \delta / \partial \Delta \phi=0$. Using the expression of the polarization orientation (Eq. (9)), the previous condition leads to $\sin (2 \alpha+\Delta \Phi)=0$. Replacing this latter result in the expression of the ellipticity (Eq. (9)), it appears that the working point that allows measuring one microwave $\mathrm{E}$ field component, while getting rid of PMF optical length variations, is characterized by a linearly polarized probe beam oriented with an angle of $45^{\circ}$ about the Wollaston prism eigen dielectric axes. For that purpose, a quarterwave plate is added between the half-wave plate and the PMF (see Fig. 4).
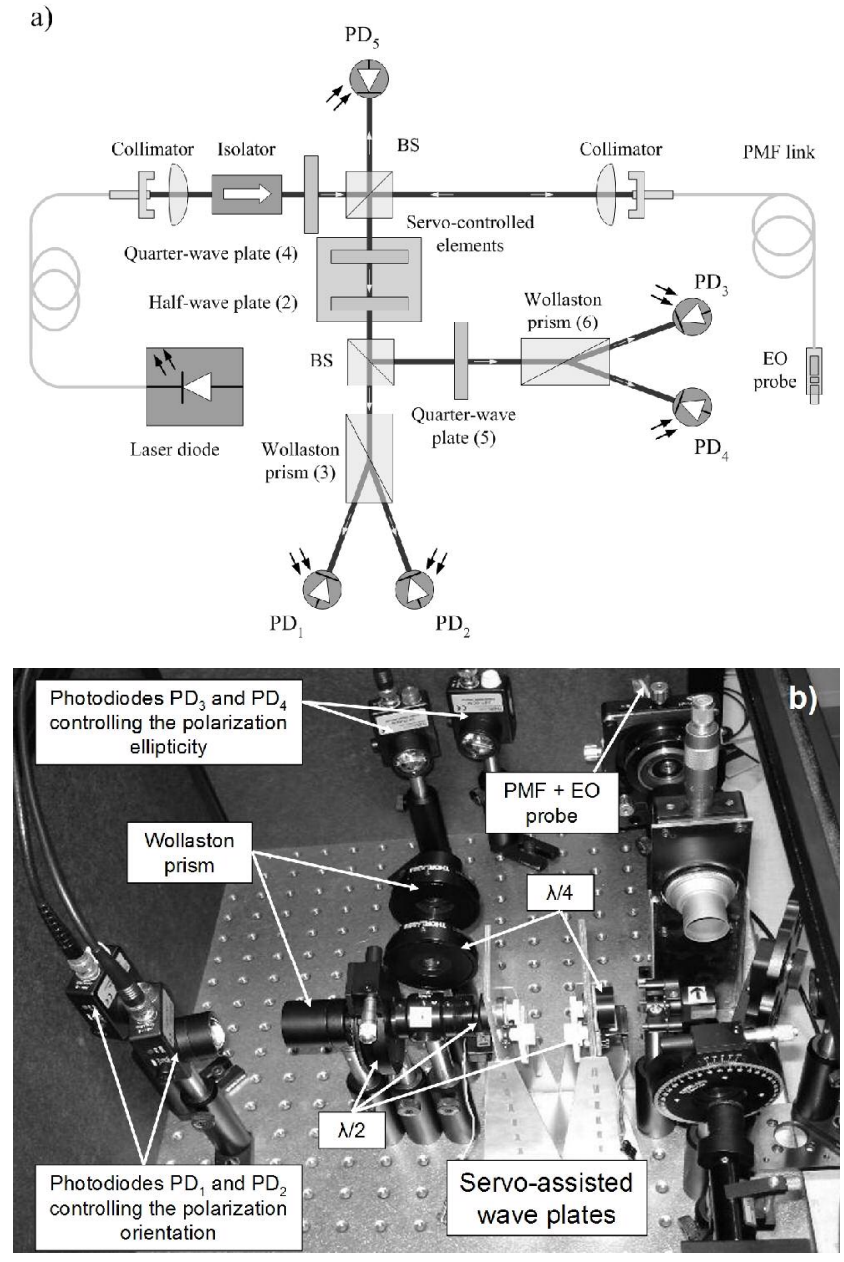

Fig. 4. a) Scheme and b) picture of the real-time servo-assisted setup for E-field measurement using EO sensors based on polarization state modulation.

Thus, in the new setup presented in Fig. 4, the laser probe beam coming out of the PMF is turned into a linearly polarized laser beam via the quarter-wave plate and is then oriented at $45^{\circ}$ about the eigen dielectric axes of the Wollaston prism (element (3) in Fig. 4) via the half-wave plate. To allow a real-time tracking of the new optimal 
working point, the two quarter- and half-wave plates are mounted on servo-controlled rotation stages.

If we consider the set of Eq. (6), the working point defined in part II is characterized by balanced optical powers $I_{1}$ and $I_{2}$ in absence of $\mathrm{E}$ field. Therefore, the compensation system, consisted of a digital PI (Proportional Integrator) regulation loop, manages to adjust the half-wave plate until the difference between the optical powers received by $\mathrm{PD}_{1}$ and $\mathrm{PD}_{2}$ is nil. A new detection path has been added to ensure getting a linearly polarized laser beam impinging on the Wollaston prism (element (3) in Fig. 4). Straightforward calculations show that the optical powers $I_{3}$ and $I_{4}$ detected by the photodiodes $\mathrm{PD}_{3}$ and $\mathrm{PD}_{4}$, respectively, are balanced for a linearly polarized probe beam at the entrance of the quarter-wave plate (element (5) in Fig. 4) as long as the eigen dielectric axes of this quarter-wave plate make an angle of $45^{\circ}$ about the Wollaston prism (element (6) in Fig. 4). Therefore, the orientation of the rotating quarter-wave plate ensures the required linearly polarized probe beam since the difference between $I_{3}$ and $I_{4}$ vanishes. The servo-controlled system we have developed consists of two independent PI regulation loops providing the two signals that control the orientation of both quarter- and half-wave plates such as the absolute values of the error functions $\varepsilon_{1}=I_{2}-I_{1}$ and $\varepsilon_{2}=I_{4}-I_{3}$ are minimized.

To sum up, this new setup allows getting rid of the temperature induced PMF birefringence variation while maintaining the highest linearity and sensitivity to any birefringence variation (linked to $\Delta \varphi$ ) of the EO crystal. Unfortunately, the relative phase shift $\Delta \varphi$ is linked at the same time to the E-field and temperature induced birefringences of the EO crystal. However, these two effects occur on very different time scales. So, via the rotation of half- and quarter-wave plates, the PI regulation loop (cut-off frequency $\sim 10 \mathrm{~Hz}$ ) permits to fully compensate for the thermal drift of the optimal working point induced by the EO crystal.

Now let us treat the already mentioned problem of the parasitic reflections at the different interfaces between the constituting elements of the probe and the PMF. These reflections lead to parasitic Fabry-Pérot cavities that lead, in turn, to variations of the return optical power $I_{0}$. Indeed, two parasitic Fabry-Pérot cavities appear in our pigtailed EO probe, the first one of optical length $L_{1}$ being formed by the two extremities of the PMF, and the second one of optical length $L_{2}$ being formed by the PMF end and the EO probe tip. Once these two Fabry-Pérot cavities taken into account, the sensitivity expression rewrites as:

$$
S=I_{0} \frac{4 m_{1} \sin ^{2}\left(\frac{2 \pi L_{1}(T)}{\lambda}\right)}{1+4 m_{1} \sin ^{2}\left(\frac{2 \pi L_{1}(T)}{\lambda}\right)} \cdot \frac{4 m_{2} \sin ^{2}\left(\frac{2 \pi L_{2}(T)}{\lambda}\right)}{1+4 m_{2} \sin ^{2}\left(\frac{2 \pi L_{2}(T)}{\lambda}\right)} \cdot \frac{4 \pi L|\Delta \vec{K}|}{\lambda}
$$

with

$$
m_{1}=\frac{4 \sqrt{R_{1} R_{2}}}{\left(1-\sqrt{R_{1} R_{2}}\right)^{2}}
$$

$R_{1}, R_{2}$ and $R_{3}$ are the reflection coefficients at the PMF input and output, and at the EO probe tip, respectively. Let us remark that the optical lengths $L_{1}$ and $L_{2}$ of the parasitic Fabry-Pérot cavities depend also on the temperature $T$ as the refractive indices of both PMF and EO crystal are temperature dependent.
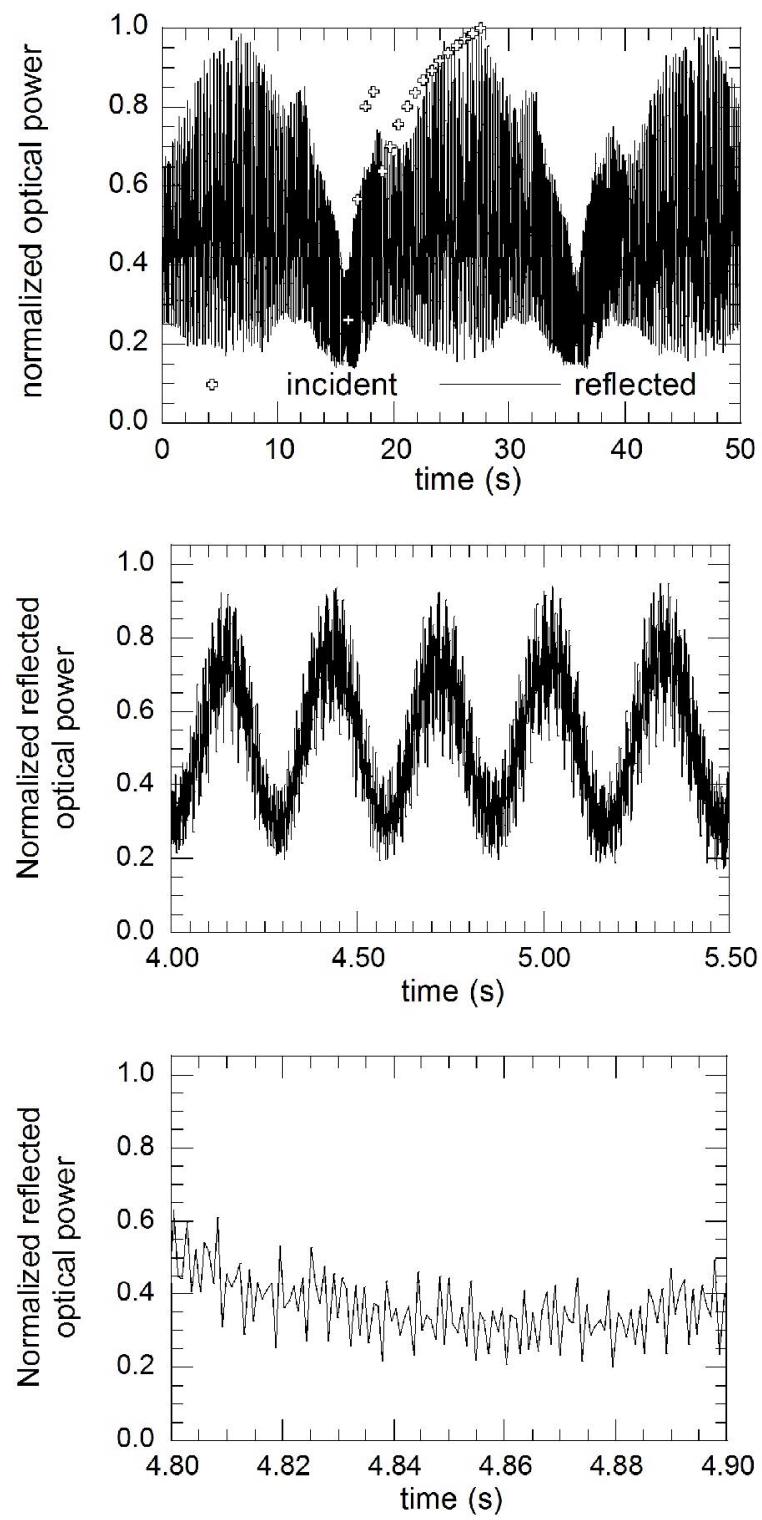

Fig. 5. Normalized optical power reflected by the pigtailed EO probe (top, solid line), zoomed in on different time scales (middle and bottom curves) and emitted by the laser diode (top curve, crosses); all curves being obtained for a triangular modulation of the laser diode temperature. 
By varying the laser diode temperature using a triangular waveform generator with a 20 -s period, the laser diode wavelength is also, to the first order, triangularly modulated (3.1 nm full scale variation). As seen on Fig. 5, the optical power reflected by the pigtailed EO probe (top curve, solid line) exhibits an envelope corresponding to the optical power emitted by the laser diode (top curve, crosses). A mode hopping occurs at time $t=18 \mathrm{~s}$. If we zoom in on the top curve, we can observe two strong modulations occurring on very different time scales (middle and bottom curves).

The strong modulation appearing in Fig. 5 leads to a strong detrimental modulation of up to $4 \mathrm{~dB}$ of the EO probe response, even for the actual reflection coefficients that are only of a few percents. Around $t=4.85 \mathrm{~s}$ (for which the wavelength variation with time is of the order of $\sim 200 \mathrm{pm} / \mathrm{s}$ ), The short and long periods ( $300 \mathrm{~ms}$ and $\sim 1.9 \mathrm{~ms})$ observed on the middle curves in Fig. 5 correspond to free spectral ranges of $\sim 7.5 \mathrm{GHz}$ and $\sim 45 \mathrm{MHz}$, respectively. From these free spectral ranges, the optical lengths $L_{1} \sim 2 \mathrm{~cm}$ (EO probe) and $L_{2} \sim 3.5 \mathrm{~m}$ (short optical PMF link used for this measurement) are deduced: they are in good agreement with the ones calculated from the physical lengths and refractive indices of the constituting components (PMF, EO crystal, ...), i.e. $L_{1}=2.85 \mathrm{~cm}$ and $L_{2}=4.5 \mathrm{~m}$ respectively.

The observed detrimental fast modulation (linked to the short period and corresponding to the long parasitic FabryPérot cavity) has been strongly attenuated using an angle polished connector (APC), as seen in Fig. 6. The modulation depth is reduced by 2 orders of magnitude, thus eliminating the detrimental effect linked to the long parasitic Fabry-Pérot cavity.

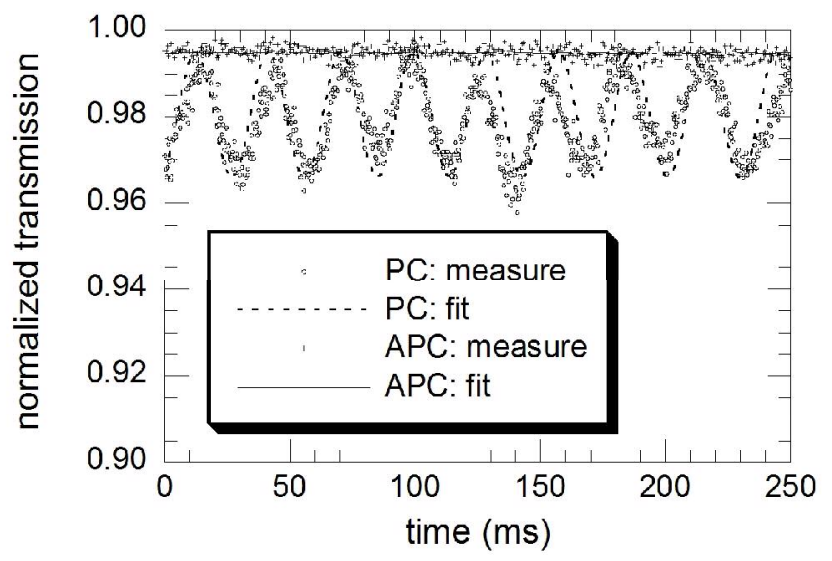

Fig. 6. Normalized optical powers transmitted through a cavity constituted by a 2-meters long PMF connected either by FC/PC or FC/APC (Angle Polished) connectors. Measurements have been made using the setup depicted in Fig. 7.

The measurements shown in Fig. 6 have been carried out using the setup depicted in Fig. 7. For these measurements, we have normalized the the optical power transmitted through the PMF by the incident power measured by the reference photodiode.

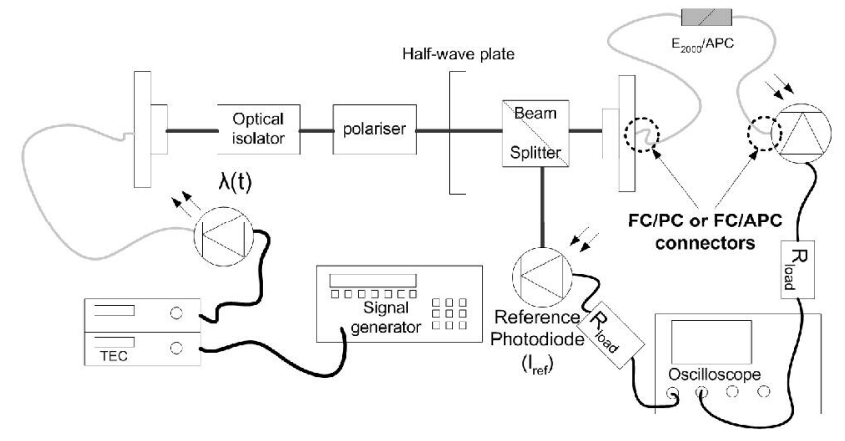

Fig. 7. Scheme of the setup used to quantify the fast modulation observed in Fig. 6.

With the use of an APC connector, we have then almost completely removed the detrimental fast modulation of the EO signal delivered by our setup. Concerning the suppression of the slow modulation, as seen in (10), a constant and maximum reflected optical power can be easily maintained by controlling the emitted wavelength. Indeed, the slow modulation, induced by the short parasitic Fabry-Pérot cavity, is real-time compensated by a third PI regulation loop which servo-control the emitted wavelength such as the derivative of the whole optical power $I_{1}+I_{2}$ (or $I_{3}+I_{4}$ ) is minimized. In addition, since the optical power emitted by the DFB laser diode depends on the wavelength (see bottom curve in Fig. 5), a fourth parameter needs to be real-time servo-controlled. For that purpose, a fifth photodiode $\mathrm{PD}_{5}$ (see Fig 4-a), is used to permanently adjust the emitted optical power to the user-defined value.

\section{EXPERIMENTAL RESULTS}

A first campaign of measurements has permitted to characterize the pulse response of the EO probe associated with the optical bench described in the previous part. Measurements have been made using a HPM pulsed source providing bursts of ten pulses at a repetition rate of $100 \mathrm{~Hz}$ with a 9-GHz carrier. Typical pulses, measured in the farfield region, are shown in Fig. 8 for which the measurements have been carried simultaneoulsy with a reference waveguide antenna and with our pigtailed EO probe.

Without the four-parameters servo-assisted system, a 0.4-dB standard deviation of the intra-burst EO signal has been observed in comparison with the reference antenna measurements (over time duration of 0.1 second). This standard deviation reaches $3.8 \mathrm{~dB}$ for inter-burst measurements which were delayed each other by several seconds. Fig. 9 shows how the addition of the real-time servo-assisted setup provides an EO rms level fluctuation lower than $0.2 \mathrm{~dB}$ over a time duration of several hundreds 
of seconds, the measurement being made using a CW HPM source.
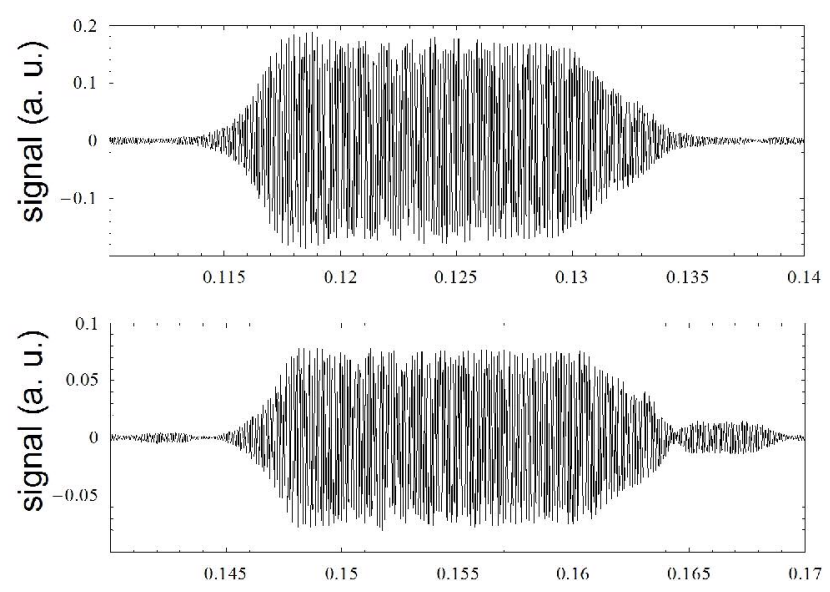

Fig. 8. Single shot high power microwave E-field measurements obtained by a reference waveguide antenna (top) and the pigtailed EO probe (bottom).

Fig. 8 exhibits a very good agreement between the signals delivered by the cylindrical shape EO probe and the reference antenna, respectively. Indeed, as shown on the simulation result in Fig. 2, the cylindrical shape EO probe presents an inner-crystal E-field whose direction is parallel to the external $\mathrm{E}$ field to be measured, thus minimizing experimental artefacts induced by E-field continuity equations at interfaces. In addition, considering a focalized optical beam (see Fig. 2), the active part of the sensor is limited to a narrow region around the propagation axis, providing an excellent spatial resolution. Since the reference waveguide antenna is metallic, it constitutes a highly disturbing vectorial probe except in very specific conditions (far field measurements). On the contrary, our fully dielectric miniature (2.8- $\mathrm{mm}$ diameter) pigtailed EO probe is almost none invasive.

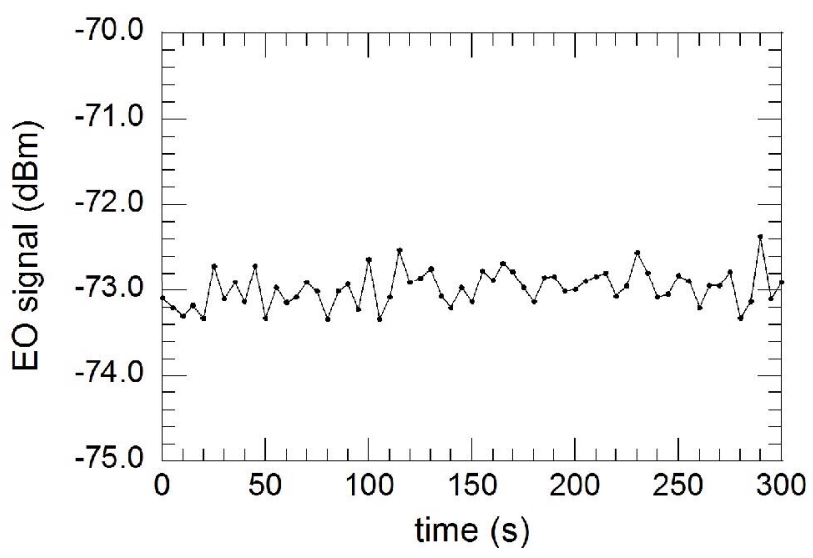

Fig. 9. Temporal evolution of the EO signal in presence of a $\mathrm{CW}$ high power microwave signal using the four-parameters servoassisted system.

Table I sums up the performances that have been obtained with the four-parameters servo-assisted system.

\begin{tabular}{|c|c|c|}
\hline $\begin{array}{c}\text { Long term } \\
\text { measurement } \\
\text { stability }\end{array}$ & $<0.2 \mathrm{~dB} \mathrm{rms}$ & $\begin{array}{c}\text { Experimental condition } \\
\text { CW high power } \\
\text { microwave, 5 minutes } \\
\text { measurement duration }\end{array}$ \\
\hline $\begin{array}{c}\text { Minimum } \\
\text { measurable E-field }\end{array}$ & $\approx 24 \mathrm{kV} \cdot \mathrm{m}^{-1}$ & $\begin{array}{c}\text { Single shot - wide } \\
\text { bandwidth }(1.13 \mathrm{GHz} \text { set } \\
\text { by a microwave amplifier) }\end{array}$ \\
\hline $\begin{array}{c}\text { Minimum } \\
\text { measurable E-field } \\
\text { (1Hz-bandwidth) }\end{array}$ & $0.7 \mathrm{~V} \cdot \mathrm{m}^{-1} \cdot \mathrm{Hz}^{-1 / 2}$ & $\mathrm{CW}$ signal \\
\hline $\begin{array}{c}\text { Modulation depth } \\
\text { of the EO signal }\end{array}$ & $4.4 \%$ & $\begin{array}{c}\text { Radiated E-field }(0-p e a k): \\
800 \mathrm{kV} \cdot \mathrm{m}^{-1}\end{array}$ \\
\hline $\begin{array}{c}\text { Selectivity (rejection } \\
\text { of orthogonal E-field } \\
\text { components) }\end{array}$ & $\geq 25 \mathrm{~dB}$ & Far field \\
\hline
\end{tabular}

Table I. Characteristics of the developed pigtailed EO probe and its associated four-parameters servo-assisted system.

\section{CONCLUSION}

The development of a real-time servo-assisted system results in a huge improvement of the EO transducer temporal stability ( $>3$ orders of magnitude). As it has been shown all along this paper, the designed probe associated with the servo-assisted optical bench, allows getting much more reliable measurements. Fig. 8 shows a single-shot measurement of an electromagnetic pulse generated in free space by a high power microwave generator, and has been obtained using a long fiber link (>20 m) under hard outdoors conditions (temperature variations up to $30^{\circ} \mathrm{C}$ ). Moreover, the handling of the system is getting much simpler since the research of the optimal working point is now automatically done by the software which drives the whole system. 


\section{ACKNOWLEDGMENTS}

The authors would like to acknowledge the DGA (French Military Programs Management and Procurement Agency) for the support.

\section{REFERENCES}

[1] M. Bernier, L. Duvillaret, G. Gaborit, A. Paupert, and J.-L. Lasserre, "Temperature-dependent-free pigtailed electro-optic sensors for vectorial measurement of microwave signals," in Proc. 6th IEEE SENSORS Conference, Atlanta, USA, pp. 573-576, 2007.

[2] J. A. Valdmanis, G. A. Mourou, and C. W. Gabel, "Subpicosecond electrical sampling", IEEE J. Quantum Electron., vol. 19, pp. 664-667, 1983.

[3] B. H. Kolner and D. M. Bloom, "Electrooptic sampling in GaAs integrated circuits," IEEE J. Quantum Electron., vol. 22, pp. 79-93, 1986.

[4] K. Yang, G. David, J.-G. Yook, I. Papapolymerou, L. P. B. Katehi, and J. F. Whitaker, "Electrooptic mapping and finite-element modeling of the near-field pattern of a microstrip patch antenna", IEEE Trans. Microwave Theory Tech., vol. 48, pp. 288-294, 2000.

[5] H. I. Bassen and G. S. Smith, "Electric field probes - A review", IEEE Trans. Antennas Propagat., vol. 31, pp. 710-718, 1983.

[6] D.-Y. Chen and J. D. Phillips, "Analysis and design optimization of electrooptic interferometric modulators for microphotonics applications," IEEE J. Ligthwave Technol., vol. 24, pp. 2340-2346, 2006.

[7] Y. Barad, Y. Lu, Z.-Y. Cheng, S.-E. Park, and Q. M. Zhang, "Composition, temperature, and crystal orientation dependence of the liner electro-optic properties of $\mathrm{Pb}\left(\mathrm{Zn}_{1 / 3} \mathrm{Nb}_{2 / 3}\right) \mathrm{O} 3-\mathrm{PbTiO}_{3}$ single crystals," Appl. Phys. Lett., vol. 77, pp. 1247-1249, 2000.

[8] M. Bernier, G. Gaborit, L. Duvillaret, A. Paupert, and J.-L. Lasserre, "Electric field and temperature measurement using ultra wide bandwidth pigtailed electro-optic probes," to appear in Appl. Opt., vol. 47, 2008.

[9] W. D. Parther, C. E. Baum, R. J. Torres, F. Sabath, D. Nitsch, "Survey of worldwide high-power wideband capabilities," IEEE Trans. Electromagn. Compat., vol. 46, pp. 335-344, 2004.

[10] L. Duvillaret, S. Riallland, and J.-L. Coutaz, "Electro-optic sensors for electric field measurements. I. Theoretical comparison among different modulation techniques," J. Opt. Soc. Am. B, vol. 19, pp. 2692-2703, 2002.

[11] B. E. A. Saleh and M. C. Teich, "Fundamentals of Photonics," Chap. 6, Wiley-Interscience, New York, 1991.

[12] G. C. Baldwin, "An Introduction to Non Linear Optics," pp. 58-60, Plenum Press, 1969.

[13] L. Duvillaret, S. Rialland, and J.-L. Coutaz, "Electro-optic sensors for electric field measurements. II. Choice of the crystals and complete optimization of their orientation," J. Opt. Soc. Am. B, vol. 19, pp. 2704-2715, 2002.

[14] A. Bruner, D. Eger, M. B. Oron, P. Blau, M. Katz, and S. Ruschin, "Temperature-dependent Sellmeier equation for the refractive index of stoichiometric lithium tantalate", Opt. Lett., vol. 28, pp. 194-196, 2003.

[15] R. B. Dyott, "Elliptical Fiber Waveguides," Chap. 6.8, Artech House, 1995.

[16] J.-P. Pérez, "Optique Fondements et Application", Chap.20, Dunod, Paris, 2004. 